\title{
Effect of pocket irrigation with antimicrobial on prevention of pacemaker pocket infection: a meta-analysis
}

Feng-Guang Kang ${ }^{1}$, Pei-Jian Liu', Li-Yi Liang ${ }^{1}$, Yong-Qing Lin²,3, Shuang-Lun Xie ${ }^{2,3}, Y_{i} \mathrm{He}^{1}$, Bao-Shan Liang ${ }^{1}$, Hai-Feng Zhang ${ }^{2,3^{*+}}$, Yang-Xin Chen ${ }^{2,3^{*+}}$ and Jing-Feng Wang ${ }^{2,3^{*+}}$ (D)

\begin{abstract}
Background: The presence of cardiac implantable electronic devices (CIEDs) pocket infection is difficult to treat, causing serious clinical outcomes, but little is known for prevention. Results from some studies suggested that pocket irrigation could reduce infection while others showed conflicting results. We pooled the effects of pocket irrigations on the prevention of pocket infection by meta-analysis methods.

Method: Relevant studies published before June, 2017 were retrieved mainly by the computer-based search of PubMed, Cochrane, EMBASE, Web of Science, Chinese BioMedical, Global Health and BIOSIS Previews databases. Estimations of relative ratios (RRs) and 95\% confidence intervals (95\% Cls) were pooled. Subgroup analyses according to potential key factors affecting the effects were conducted, which was confirmed by meta-regression. Sensitivity analysis and test for publication bias were also performed.

Results: We identified 10 studies providing data of 5467 patients receiving CIEDs implantations. Pooled infection rates were 1.48 and 3.49\% respectively for medication and saline irrigation groups. Meta-analysis showed that medication irrigation conferred protection to pocket infection ( $R R=0.44,95 \% \mathrm{Cl}$ : 0.31-0.63). Subgroup analysis showed that antibiotics, rather than non-antibiotics (antiseptics) exerting the protection. The first and second lines antibiotics against staphylococcus aureus, which is the main pathogen for pocket infection, were both effective ( $R R=0.42,95 \% \mathrm{Cl}: 0.24-0.75$ and $\mathrm{RR}=0.34,95 \% \mathrm{Cl}: 0.20-0.58$ respectively for first line and second line therapies). Meta-regression revealed that region and class of irrigation medication completely explained the variance among studies and implied that effects of region were masked by medication types. Sensitivity analysis did not showed any significant change of the result and publication bias were not statistical significance.
\end{abstract}

Conclusion: Pocket irrigation with antibiotics were effective for reducing pocket infection and should be encouraged in CIEDs implantation.

Keywords: Cardiac implantable electronic devices, Pocket irrigation, Pocket Infection, Meta-analysis

\section{Background}

Cardiac implantable electronic devices (CIEDs) mainly include pacemaker, implantable cardiac defibrillator (ICD), cardiac resynchronization therapy (CRT). Of these, pacemakers are the most common and effective way to treat bradycardia arrhythmia (pacemaker),

\footnotetext{
*Correspondence: zhanghf9@mail.sysu.edu.cn; tjcyx1995@163.com; dr_wjf@sina.com

${ }^{\dagger}$ Equal contributors

${ }^{2}$ Department of Cardiology, Sun Yat-sen Memorial Hospital, Sun Yat-sen University, Guangzhou, Guangdong 510120, People's Republic of China Full list of author information is available at the end of the article
}

while ICD and CRT are effective strategies to prevent sudden death and improve heart failure, respectively. The number of CIEDs implantations have increased significantly during the past decade. There were 560,000 CIEDs implanted in the United States every year $[1,2]$. Accordingly, the number of infectious complications attributed CIEDs implantation also increased dramatically $[1,3]$. Subcutaneous pocket infection, which is mainly due to staphylococcus aureus [4], is the most serious infectious complications $[5,6]$. The reported incidence of these CIEDs-related 
infections has been ranging from 0 to $12.6 \%$ in studies $[3,7,8]$, mostly ranging from 1 to $7 \%[9]$.

Among all the complications, pacemaker/ICD/CRT pocket infection remains a serious, and even, potentially life-threatening complication [8]. Once pocket infection occurred, it will prolong hospital stay, increase medical cost, especially when a removal of the entire system with subsequent re-implantation is needed [10-12]. Moreover, the increased incidence of infectious complication is associated with substantial elevated morbidity and mortality $[8,13,14]$.

Although there are data supporting systemic use of antibiotic for preventing infection during and after CIEDs implantations, many employ other accompanying strategies for further prevention $[2,15]$. One of the strategy is pocket irrigation with antimicrobial solutions, although there is no conclusive evidence demonstrating its benefit. Indeed, results from individual trials were conflicting and not convincing. For example, results from Lakkireddy and colleagues showing an infructuous effect of pocket irrigation while other claimed a reduced rate of infection $[16,17]$. To summary current evidence and draw a plausible conclusion, we performed a meta-analysis of available trials to re-evaluate the effectiveness of pocket irrigation with antimicrobial agents in reducing pocket infection during CIEDs implantation.

\section{Methods}

\section{Literature search and study selection}

All studies reporting the effects of pocket irrigation during CIEDs implantation published before June 2017 were identified by the comprehensive computer-based search of PubMed, Cochrane, EmBase, Web of Science, Chinese BioMedical, Global Health and BIOSIS Previews databases. The following terms were used for search: pacemaker, cardiac implantable device, VVI, DDD, implanted cardiac defibrillator, ICD, cardiac re-synchronization therapy and CRT, which were combined with pocket, irrigation, and infection. Hand searches for related articles were also performed. All the searches were conducted without language restriction. Reference lists of the retrieved articles were also reviewed to ensure to no eligible study missed.

For inclusion, procedure of the implantation had to be described properly to ensure that no operative factors leading to infection. Exclusion criteria were as follows: 1) no recording infectious rates between groups; 2) non-medication treatments of the pocket; 3 ) including pericardial lead implantation; and 4) sample size less than 100.

\section{Data extraction and quality assessment}

Two investigators (Kang FG and Zhang HF) extracted data independently. All the data were extracted using a standardized data-collection form. Information was recorded as follows: last name of the first author, year of publication, geographical location, study design, agents for irrigation, time from implantation to infection, duration of the followup period and number of participants.

The quality of enrolled studies were also assessed by the same investigators and the following elements were considered [18]: study design, characteristics of the studied population, assessment of outcome, duration of follow-up, and statistical control for potential confounding factors. Any disagreement were resolved by a discussion. All the data were extracted from published results and written informed consent for participation was not applicable.

\section{Statistical analysis}

Meta-analysis was performed as our previous report $[19,20]$. In brief, heterogeneity of effect size across studies was quantified by the $I^{2}$-statistic and tested by a Cochrane $Q$-test with a significance level of $P<0.1$, rather than 0.05 [21]. Pooled effect size was estimated by Mantel-Haenszel fixed-effects model if no significant heterogeneity existed. Otherwise, the DerSimonian-Laird random-effects model was adopted. Potential publication bias was assessed by Egger's test and Begg's funnel plot was produced [22].

To further investigate the effects of pocket irrigation during CIEDs implantations, subgroup analyses according to study designs, classes of medications, time from implantation to infection, and geographical locations were performed. Meta-regression using restricted maximum likelihood estimation method was adopted to explore sources of heterogeneity and to confirm results from subgroup analyses [23]. $P$-values were adjusted with the Monte Carlo method (permute $=100$ ) if more than 3 co-variables enrolled in the regression model to reduce type I error [24]. Finally, a sensitivity analysis, which investigated the influence of a single study on the overall risk estimated by omitting one study in each turn, was used to test the stability of the pooled results.

The study was performed in accordance with the PRISMA statement [25]. All analyses were performed by using STATA version 12.0 and graph of quality assessment was produced by Revman 5.3 .

\section{Results \\ Eligible studies}

With separated search strategy in each database, a total of 433 articles that potentially pertinent were retrieved. By reviewing titles and abstracts, irrelevant studies, case reports and reviews were excluded. Finally, 13 studies were identified for further considerations. Of the these, 3 studies were further excluded mainly due to an absence of control group (two studies) and comparing short term and long term usage of antibiotics (one 
study). Therefore, ten studies including 5467 patients (3117 in irrigation group and 2340 in control group) completely met the inclusion criteria, which were used in the later analysis $[16,17,26-33]$. The flow diagram of searching and screening publications were listed in Fig. 1.

\section{Study characteristics}

Characteristics of the included studies were presented in the Table 1. Overall, enrolled trials were published from 2003 to 2017, including American and Chinese studies, and ranging from 118 to 2654 in sample size. Most of the studies used antibiotics and one study used povidone-iodine to irrigate the pocket [16]. Of the trials using antibiotics, the third generation of cephalosporin was used in three studies, dcefazolin and gentamicin were used in two studies, clindamycin and azithromycin were used in only one study. Besides, povidone-iodine was used in one study (Table 1).
Two studies were retrospective in design and the rest were prospective ones (Table 1).

All the included studies were low in detection and attrition bias, while most studies were with low in attrition bias and more than a half of the studies were with low to unclear bias in selection, performance and other bias (Fig. 2).

\section{Main analyses \\ Pocket irrigation with anti-biotics was effective to reduce pocket infection}

Overall, pocket infection rate was $1.48 \%(46 / 3117)$ in the irrigation group and $3.49 \%(82 / 2350)$ among the controls. Pooled analysis including all the studies was firstly performed and results from fixed-effects model showing a beneficial effect of pocket irrigation, reducing 56\% infectious rate $(\mathrm{OR}=0.44,95 \% \mathrm{CI}$ : 0.31 to 0.63 , Fig. 3$)$. Mild to moderate heterogeneity was observed among

433 Articles were retrieved for inclusion

67 PUBMED/ 147 Embase

13 Cochrane database/ 5 Web of Science 112 Global Health/ 22 BIOSIS Preview 62 Chinese BioMedical/5 Hand search

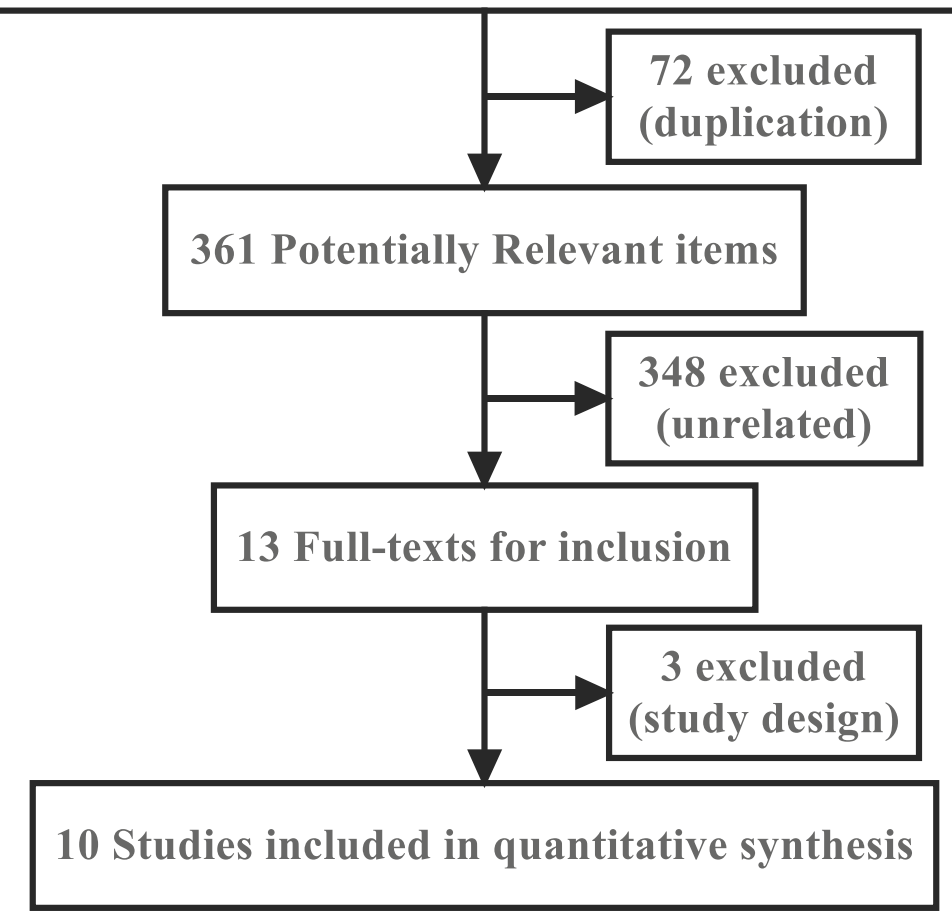

Fig. 1 Systematic search and screening process of included trials 
Table 1 Characteristics of the included studies

\begin{tabular}{|c|c|c|c|c|c|c|c|c|}
\hline Study & Location & Study design & $\begin{array}{l}\text { Gender } \\
\text { ( } n \text {, male) }\end{array}$ & Agent & $\begin{array}{l}\text { Follow-up } \\
\text { period }\end{array}$ & Samle size & $\begin{array}{l}\text { Treatment } \\
\text { Infection/Total }\end{array}$ & $\begin{array}{l}\text { Control } \\
\text { Infection/Tota }\end{array}$ \\
\hline Lu et al. 2003 [28] & China & Prosepctive & 72 & 3rd cephalosporin & 7 years & 118 & $0 / 61$ & $0 / 57$ \\
\hline Guo et al. 2005 [27] & China & Prosepctive & 101 & Cefazolin & 2 years & 178 & $2 / 90$ & $0 / 88$ \\
\hline Lakkireddy et al. 2005 [16] & America & Retrospective & 1718 & Povidone-iodine & 8 years & 2564 & $10 / 1359$ & $8 / 1205$ \\
\hline Xia et al. 2007 [29] & China & Prosepctive & 66 & Gentamicin & 3 years & 122 & $1 / 63$ & $1 / 59$ \\
\hline Zhou et al. 2010 [32] & China & Prosepctive & 155 & $\begin{array}{l}\text { Cefoperazone/ } \\
\text { Tazobactam }\end{array}$ & 1 month & 268 & $2 / 84$ & $9 / 84$ \\
\hline Wang et al. 2015 [17] & China & Prosepctive & 70 & Cefazolin & $>4$ weeks & 116 & $2 / 58$ & $8 / 58$ \\
\hline Yang 2015 [30] & China & Retrospective & 931 & Amikacin/ Gentamicin & 7 years & 1572 & $15 / 1133$ & $16 / 439$ \\
\hline Zhang et al. 2016 [31] & China & Prosepctive & 77 & Cefatriaxone & 6 months & 146 & $5 / 73$ & $21 / 73$ \\
\hline Lakshmanadoss et al. 2016 [33] & America & Retrospective & 134 & Clindamycin phosphate & 1 year & 327 & $2 / 118$ & $2 / 209$ \\
\hline Chen et al. 2017 [26] & China & Prosepctive & 95 & Azithromycin & 1 year & 156 & $7 / 78$ & $17 / 78$ \\
\hline
\end{tabular}

studies $\left(I^{2}=32.7 \%\right.$ and $Q$-test $\left.P=0.16\right)$. This result showed that the initial analysis support a protective role of pocket irrigation using medications.

Despite the above analysis did not yield much heterogeneity, we continued to perform subgroup analysis to further investigate factors that may influence the magnitude of effect size. Subgroup analysis results were presented in Fig. 4. As indicated, the protective effects of pocket irrigation were dismissed in American studies and non-antibiotics cohorts (Fig. $4 \mathrm{a}$ and b). Similarly, results from prospective trials differed from those of retrospective ones (Fig. 4c). Both first line and second line antibiotics therapies against staphylococcus aureus, which is the leading pathogen of pocket infection, were effective but more benefits were observed in sencond line therapy (Fig. 4d). Pocket irrigation reduced both early (within 1 month) and late (longer than 1 month) infections, but with a slightly larger effect size in protecting early infection (Fig. 4e). All these results confirmed the protective role of antibiotics, irrespective of class of medications.

\section{Irrigation with non-antibiotics did not reduce pocket infection}

The above analyses found that results from the American cohort (two studies), retrospetive cohort (three studies) and non-antibiotics cohort (one study) did not support the protective role of pocket irrigation. Notably, there was a same study using non-antibiotics in all of these cohorts.

To confirm the above results from subgroup analyses, we performed meta-regression to seek the potential co-variables on protective effects conferred by irrigation. Meta-regressions using mean age, region, study design, including patients receiving ICD (or CRT) or not, antibiotics/non-antibiotics, first/second line therapy against staphylococcus aureus, and early/ late infections as co-variables independently were done. Results indicated that age, study design, including CRT or ICD patients, first/second line therapy for staphylococcus aureus, or early/late infections were not the source of heterogeneity (mean age: $P=0.34$; study design: $P=0.24$; CRT or ICD included: $P=0.72$; first/second line therapy for staphylococcus aureus: $P$

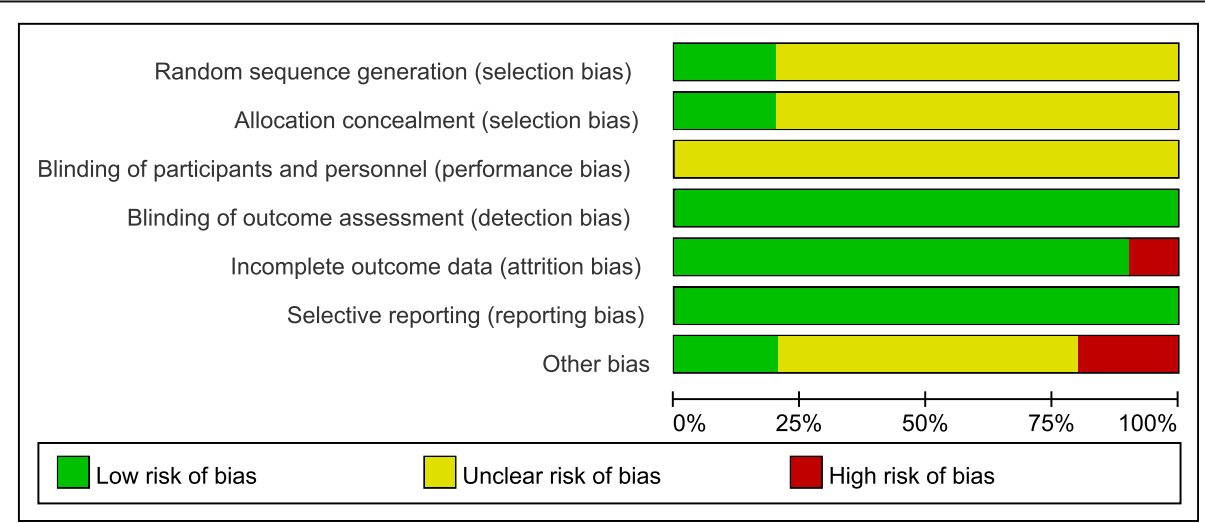

Fig. 2 Quality assessments of included studies 


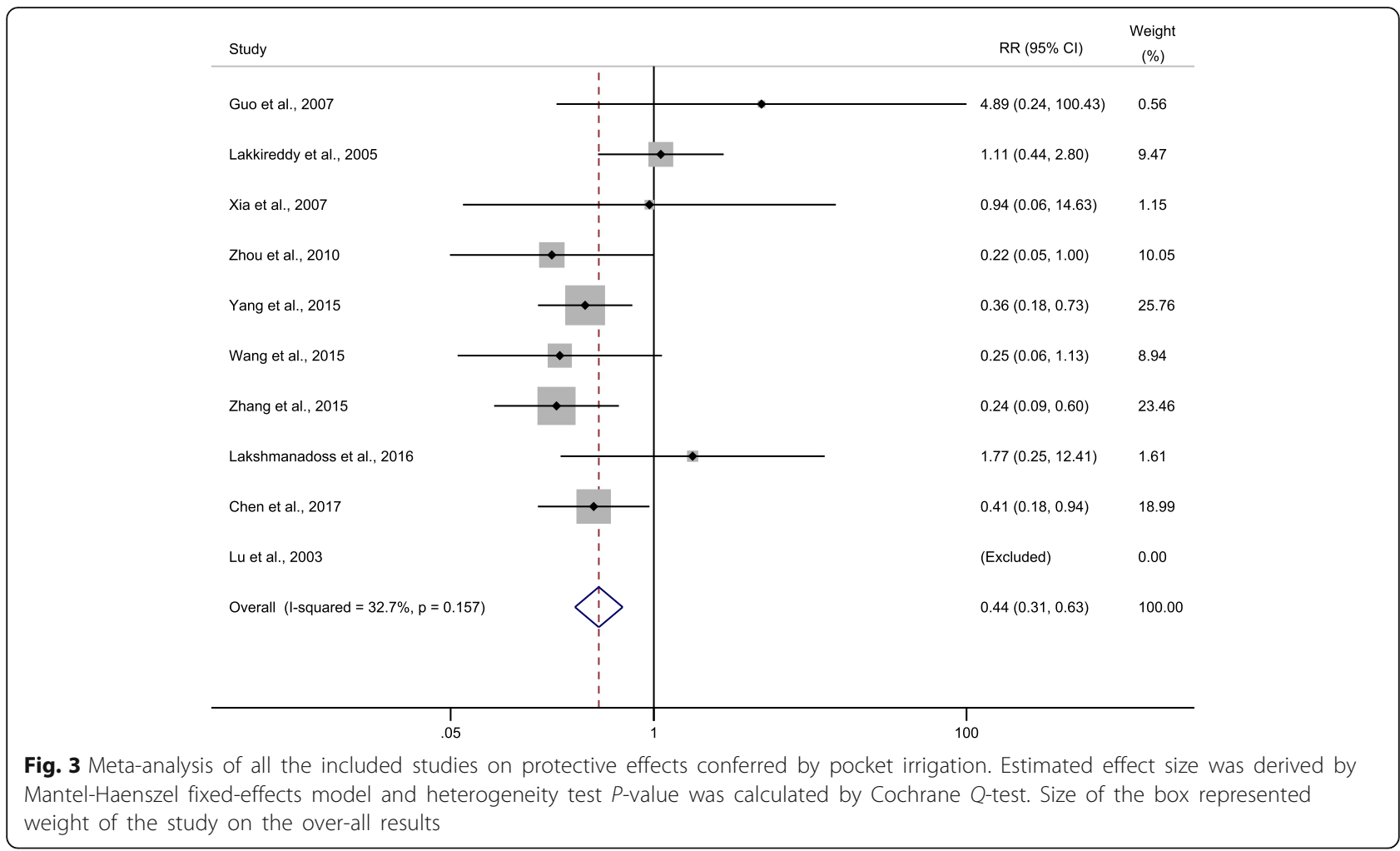

$=0.18$; early/late infections: $P=0.33$ ). However, both region and antibiotics/non-antibiotic may account for heterogenetity (region: $P=0.03$; antibiotics/non-antibiotics: $P=0.08)$. Both of the two co-variables completely account for the intra-studies variance (both adjusted $R_{\text {meta }}^{2}=100 \%$ ). Because one of the study using non-antibiotics was the American one [16], we therefore further generated an interaction variable between region and antibiotics/non-antibiotics used, which was later used in meta-regression. Results revealed that effects of this interaction (region and antibiotics/nonantibiotics) were exactly the same as using nonantibiotics alone as co-variable (both regressions OR $=2.99, P=0.08$, Fig. 5). Therefore, the meta-regression results implied that the significant influence manifested by region may be actually masked by antibiotics/non-antibiotics used in studies.

\section{Sensitivity and publication bias}

Furthermore, we performed sensitivity analysis to examine the intensity of the conclusion and results found that the protective effects of pocket irrigation were not influenced, which remained to be significant omitting any of the included study. These results implied that the protective effects of pocket irrigation were stable and robust. Publication bias determined by Begg's test did not showed a significant bias (Kendall's Score $=12, P=0.21$, Fig. 6).

\section{Discussion}

Pocket infection is the most serious complication of CIEDs implantation and current evidence on the effects of pocket irrigation is inconsistent. To summary available information in this field, the meta-analysis of 10 studies involving 5467 participants supported a protective effect of antibiotics pocket irrigation, irrespective of antibiotics classes. Compared with the use of saline, the incidence of pocket infection was reduced by about $59 \%$ with antibiotic irrigation.

Implantations of CIEDs have increased over the past decades, despite implantation technique improvement, the infection rate has increased dramatically $[2,8]$. Pocket infection may be due to the following causes [34]: (1) Pacing leads and pulse generators are exposed to the air before implantation, which may cause airborne pathogens; (2) To the human body, the implant is a metallic foreign body which has a rough surface, making it prone to bacteria growth; (3) Operation duration, which is greatly affected by the individual condition of the patient and the technique of the operator; (4) The ambient air quality in the operating room, which may not be sufficient to reach the real sterile in some time; 


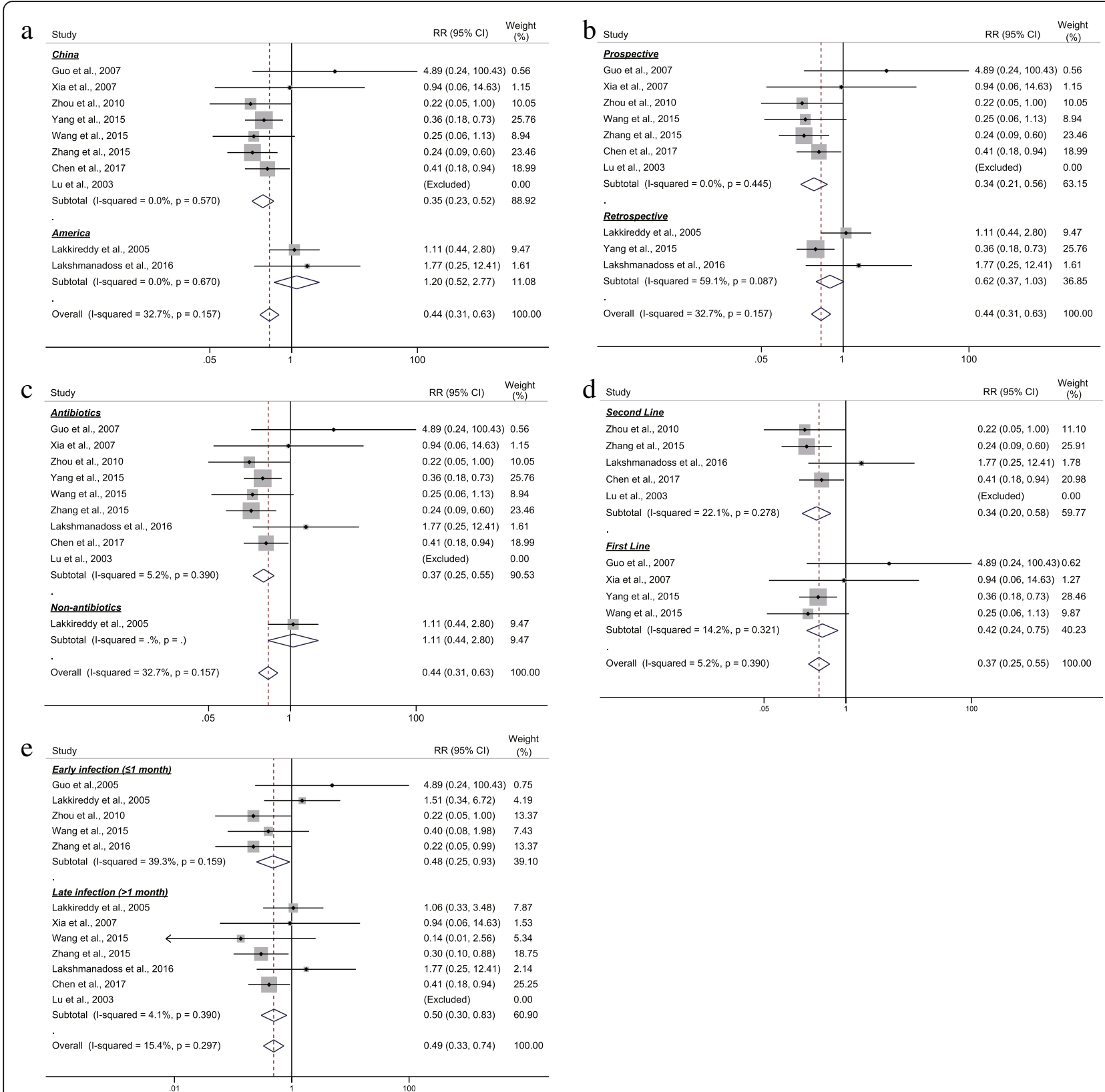

Fig. 4 Subgroup analyses according to region (a), irrigation medications (b), study design (c), and first/second line therapy for staphylococcus aureus (d) and early/late infection (e). Methods used and meaning of symbols were the same as Fig. 3

(5) Patients receiving CIEDs implantation are mostly aged ones, who could have various co-existing diseases, making them more prone to be infected. It is obvious that most of the reasons could not be completely avoided and strategies to prevent pocket infect becoming crucial.

Besides systematic application of antibiotics, little effective strategy is known to reduce pocket infection. Therefore, screening and confirming positive methods to reduce pocket infection is of great importance. Pocket irrigation may be a promising strategy for reducing pocket infection but debate on this issue is persistent during the decade $[7,35]$. Indeed, pocket infection, despite increasing, is not with high prevalence, which result in a need of large sample size to give a statistical significance. It may be difficult for a single study to reach such a large sample size. Because of the inconsistent results of the trials, current guideline did not give a recommendation on pocket irrigation to reduce pocket infection [36]. Therefore, pooled analysis of existing information in this field becoming 


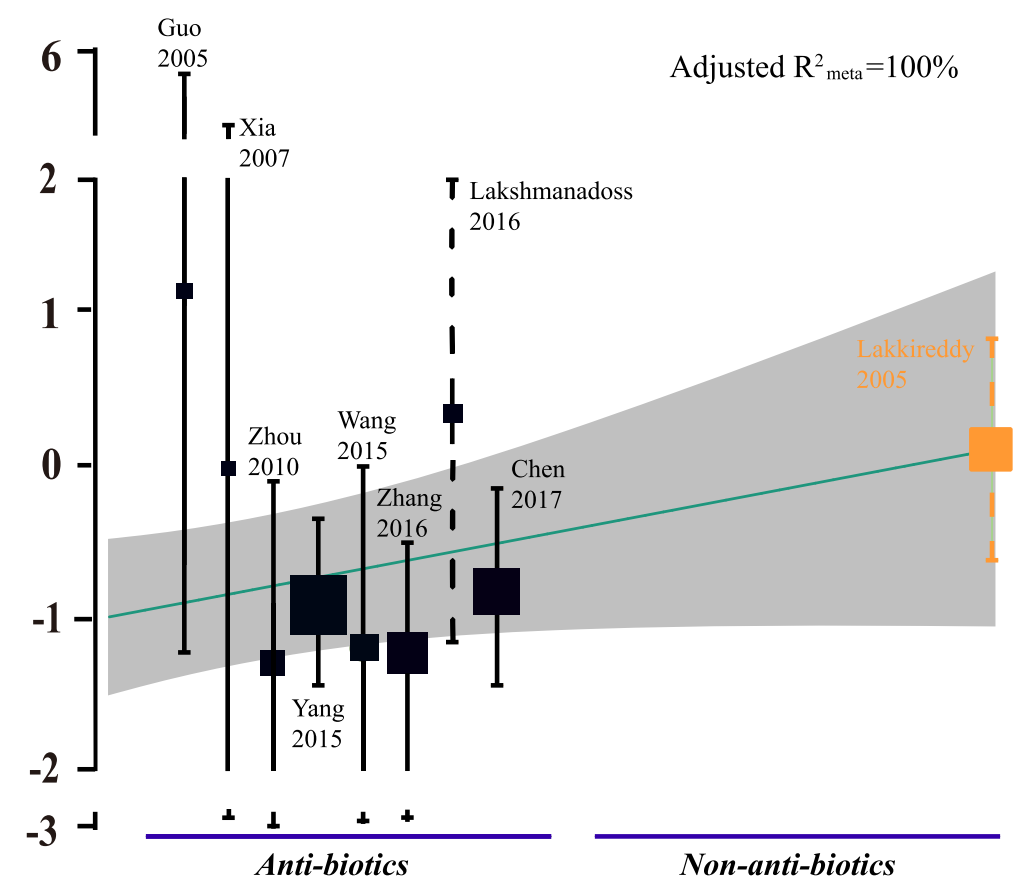

Fig. 5 Meta-regression using interaction between region and class of medication (antibiotics/non-antibiotics) used for irrigation. The interaction explained almost all of the variance among enrolled studies. Meta-analysis was performed using restricted maximum likelihood estimation. Size of the box represented study weight. Symbols represents indicated RRs and 95\% Cls of the study and dotted line indicated the American study.

Orange symbols indicated study using non-antibiotics (povidone-iodine). Gray shading area represented 95\% Cls of the regression line

important and emergent. The current study pooled all the data on pocket irrigation and showed a benefit of reducing infection, irrespective of class of antibiotics, which robustly supported that the use of antibiotics for pocket irrigation should be encouraged. Indeed, an survey of 2092 electrophysiologists in more than fifty countries believed that the use of anti-microbial agents for pocket irrigation could reduce infection of CIEDs implantation [35]. Thus, the current study provide conclusive evidence supporting this view point.

The same important is to identify the most effective drugs for irrigation. As known, the most common bacteria causing pocket infection is staphylococcus aureus [12, 36], which accounts for about 60 to $80 \%$ of the pathogens in infection [6]. For the treatment of staphylococcus aureus, cefazolin and gentamicin were recognized as the first line

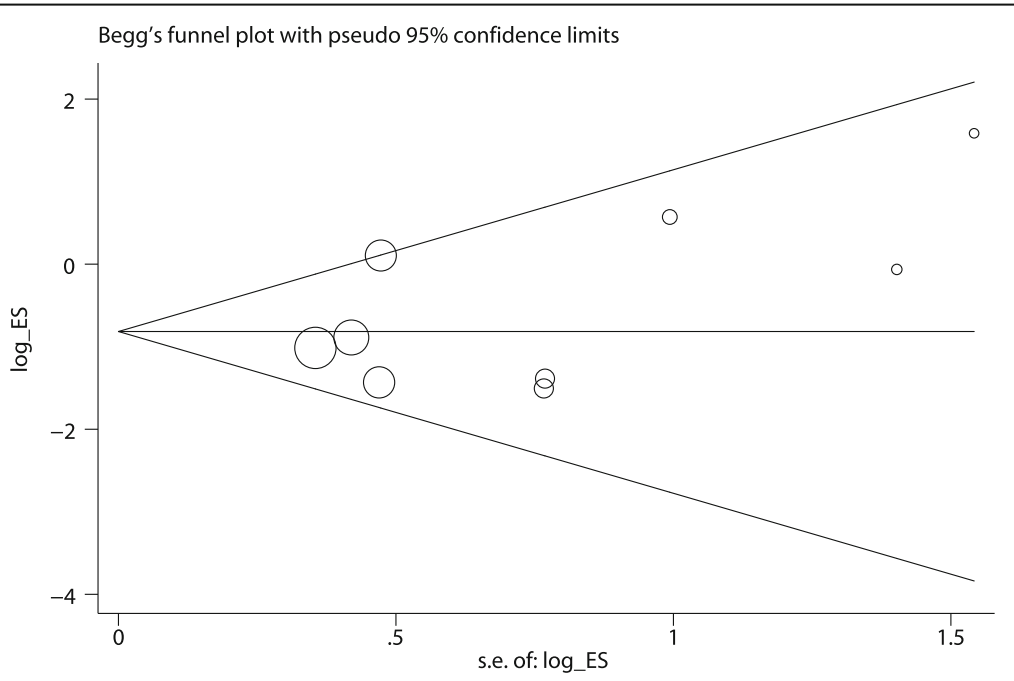

Fig. 6 Publication bias of included studies. P-value was derived by Egger's test. Size of the circle indicated study weight 
therapy while others were believed as the second line [6]. As indicated in the present study, the second line therapy for staphylococcus aureus was more effetive despite the first line therapy also conferred protective effects. Therefore, when choosing the medication for pocket irrigation for whom are proning to be infected, drugs of the second line therapy may be considered first. Besides, drug resistance of the bacteria in their own center should also be taken into consideration. For example, in south of China, cefazolin is the most frequently used while the third generation of cephalosporin and lincosamide were adopted in north of China and the USA respectively [31, 33].

Some limitations of the current study should be noted. Firstly, despite subgroup analysis of RCTs/non-RCTs also showed an ineffectiveness of retrospective studies, meta-regression did not support study design as the variable for variance. Of note, one of the retrospective study using non-antibiotics, which was shown to be the source of the heterogeneity. Therefore, it is unknown whether the retrospective study did not support the adoption of pocket irrigation in nature, or like covariable of region, which is actually masked by the use of non-antibiotics in the retrospective study. Secondly, insufficient data restricted further analysis according to different kinds of CIEDs (VVI/DDD/ICD/CRT/CRT-D) implantations, which might also be an important issue since patients receiving ICD/CRT/CRT-D were largely heart failure or ventricular arrhythmia survivors, who may be much more complicated and have distinct properties concerning with pocket infection.

\section{Conclusion}

The current study demonstrated significantly protective effect on preventing pacemaker pocket infection with antibiotics pocket irrigation, irrespectively of classes of antibiotics, which robustly supported regular use of antibiotics for pocket irrigation during CIEDs procedure. As well as a need for large sample size confirming this conclusion in prospective studies with well control of bias, there is also an urgent need for screening of most effective medication, which together contribute to the reduction of pocket infection.

\section{Abbreviations}

Cl: Confidence interval; CIEDs: Cardiac implantable electronic devices; CRT: Cardiac resynchronisation therapy; CRT-D: Cardiac resynchronisation therapy with a defibrillator; ICD: Implantable cardiac defibrillator; RR: Relative ratio

\section{Acknowledgements}

Not applicable.

\section{Funding}

This work is supported by the National Natural Science Foundation for Young Scientists of China to Zhang HF (Grant No.81300071), Natural Science Foundation of Guangdong Province of China to Lin YQ (Grant No.2014A030313049); Science and Technology Program of Guangzhou of China to Zhang HF (Grant No. 201510010048) and Wang JF (Grant No. 2014Y2-00118), and the Fundamental Research Funds for the Central Universities to Zhang HF (Grant No. 13ykpy30).

\section{Availability of data and materials}

The datasets used and/or analyzed during the current study are available from the corresponding authors (WJF) on reasonable request.

\section{Authors' contributions}

WJF, CYX and ZHF designed the study. KFG and ZHF collected the study, assessed the studies' quality, and performed the main analyses. LPJ, LLY, LYQ, $X S L, H Y$ and LBS corrected the collected data. KFG, ZHF and CYX completed the the manuscript. All authors read and approved the final manuscript.

Ethics approval and consent to participate

Not applicable.

\section{Consent for publication}

Not applicable.

\section{Competing interests}

The authors declare that they have no competing interests.

\section{Publisher's Note}

Springer Nature remains neutral with regard to jurisdictional claims in published maps and institutional affiliations.

\section{Author details}

${ }^{1}$ ShunDe Hospital of Guangzhou University of Chinese Medicine, Foshan, Guangdong 528333, People's Republic of China. ²Department of Cardiology, Sun Yat-sen Memorial Hospital, Sun Yat-sen University, Guangzhou, Guangdong 510120, People's Republic of China. ${ }^{3}$ Laboratory of Cardiac Electrophysiology and Arrhythmia in Guangdong Province, Guangzhou, Guangdong 510120, People's Republic of China.

Received: 24 June 2017 Accepted: 19 September 2017

Published online: 30 September 2017

\section{References}

1. de Oliveira JC, Martinelli M, Nishioka SA, Varejão T, Uipe D, Pedrosa AA, et al. Efficacy of antibiotic prophylaxis before the implantation of pacemakers and cardioverter-defibrillators: results of a large, prospective, randomized, double-blinded, placebo-controlled trial. Circ Arrhythm Electrophysiol. 2009; 2(1):29-34.

2. Tarakji KG, Wilkoff BL. Management of cardiac implantable electronic device infections: the challenges of understanding the scope of the problem and its associated mortality. Expert Rev Cardiovasc Ther. 2013;11(5):607-16.

3. Cabell CH, Heidenreich PA, Chu VH, Moore CM, Stryjewski ME, Corey GR, et al. Increasing rates of cardiac device infections among Medicare beneficiaries: 1990-1999. Am Heart J. 2004;147(4):582-6.

4. Schwartzman D, Pasculle AW, Ceceris KD, Smith JD, Weiss LE, Campbell PG. An off-the-shelf plasma-based material to prevent pacemaker pocket infection. Biomaterials. 2015:60:1-8.

5. Baddour LM, Cha YM, Wilson WR. Clinical practice. Infections of cardiovascular implantable electronic devices. N Engl J Med. 2012;367(9):842-9.

6. Bloom HL, Constantin L, Dan D, De Lurgio DB, El-Chami M, Ganz LI, et al. Implantation success and infection in cardiovascular implantable electronic device procedures utilizing an antibacterial envelope. Pacing Clin Electrophysiol. 2011;34(2):133-42.

7. Chua JD, Wilkoff BL, Lee I, Juratli N, Longworth DL, Gordon SM. Diagnosis and management of infections involving implantable electrophysiologic cardiac devices. Ann Intern Med. 2000;133(8):604-8.

8. Ra'Ad T, Elena D, Kasra M, Monali P, Victor M. In-Hospital mortality among patients receiving an implantable cardioverter-defibrillator in the United States: 2000 to 2012. J Am Coll Cardiol. 2016;67(13):862.

9. Karchmer AW, Longworth DL. Infections of intracardiac devices. Cardiol Clin. 2003;21(2):253-71

10. Baddour LM, Epstein AE, Erickson CC, Knight BP, Levison ME, Lockhart PB, et al. A summary of the update on cardiovascular implantable electronic device infections and their management: a scientific statement from the American Heart Association. J Am Dent Assoc. 2011;142(2):159-65.

11. Voigt A, Shalaby A, Saba S. Rising rates of cardiac rhythm management device infections in the United States: 1996 through 2003. J Am Coll Cardiol. 2006:48(3):590-1. 
12. Chu VH, Crosslin DR, Friedman JY, Reed SD, Cabell CH, Griffiths RI, et al. Staphylococcus aureus bacteremia in patients with prosthetic devices: costs and outcomes. Am J Med. 2005;118(12):1416.

13. Patricia K, Steven P. Implantable Cardioverter Defibrillator Complications: Infection, Erosion, Thrombosis. Card Electrophysiol Rev. 2001;5(1):110-4.

14. Kew LK, Fontecchio S. Infections Associated with Implantable Cardioverter Defibrillators Placed Transvenously and via Thoracotomies: Epidemiology, Infection Control, and Management. Clin Infect Dis. 1998;27(9):265-9.

15. Mela T, McGovern BA, Garan H, Vlahakes GJ, Torchiana DF, Ruskin J, et al. Long-term infection rates associated with the pectoral versus abdominal approach to cardioverter- defibrillator implants. Am J Cardiol. 2001;88(7): 750-3.

16. Lakkireddy D, Valasareddi S, Ryschon K, Basarkodu K, Rovang K, Mohiuddin SM, et al. The impact of povidone-iodine pocket irrigation use on pacemaker and defibrillator infections. Pacing Clin Electrophysiol. 2005;28(8):789-4.

17. Wang HJ, Yu SJ, Wang HL. Clinical effect of antibiotic prophylaxis on postoperative infections in elderly patients undergoing implantation of permanet pacemaker. Chin J Nosocomiol. 2015;13:2976-8.

18. Armijo-Olivo S, Stiles CR, Hagen NA, Biondo PD, Cummings GG. Assessment of study quality for systematic reviews: a comparison of the Cochrane Collaboration Risk of Bias Tool and the Effective Public Health Practice Project Quality Assessment Tool: methodological research. J Eval Clin Pract. 2012;18(1):12.

19. Zhang HF, Xie SL, Wang JF, Chen YX, Wang Y, Huang TC. Tumor necrosis factor-alpha G-308A gene polymorphism and coronary heart disease susceptibility: an updated meta-analysis. Thromb Res. 2011;127(5):400-5.

20. Zhang HF, Zhong BL, Zhu WL, Xie SL, Qiu LX, Zhu LG, et al. CD14 C-260T gene polymorphism and ischemic heart disease susceptibility: a HuGE review and meta-analysis. Genet Med. 2009;11(6):403-8.

21. Higgins JP, Thompson SG. Quantifying heterogeneity in a meta-analysis. Stat Med. 2002;21(11):1539-8.

22. Egger M, Davey SG, Schneider M, Minder C. Bias in meta-analysis detected by a simple, graphical test. BMJ. 1997;315(7109):629-34.

23. Luebke T, Brunkwall J. Meta- analysis and meta-regression analysis of the associations between sex and the operative outcomes of carotid endarterectomy. BMC Cardiovasc Disord. 2015;15:32.

24. López-López JA, Van den Noortgate W, Tanner-Smith EE, Wilson SJ, Lipsey MW. Assessing meta-regression methods for examining moderator relationships with dependent effect sizes: A Monte Carlo simulation. Res Synth Methods. 2017;

25. Moher D, Liberati A, Tetzlaff J. Altman DG; PRISMA Group. Preferred reporting items for systematic reviews and meta-analyses: the PRISMA statement. Int J Surg. 2010;8(5):336-41.

26. Chen Y, Shi BF, Chen J, Cheng HL, LV Y. Interventional effect of intraoperative antibiotics rinse on inflammatory indices, postoperative capsular infection in patients implanted with permanet pacemaker. Chin J Nosocomiol. 2017;27(9):2028-31.

27. Guo JF, Bao ZH, Li WD, Zhang GH, Zhen Y, Tang LC. Effects of pocket irrigation using antibiotics during pacemaker implantation on pocket infection. J Qiqihar Med Coll. 2005;26(11):1295-6.

28. Lu CY, Wang SW, Hu TH, Wei X, Li YF, Liu LL. Effect of antibiotic solution flushing on prevention of packmaker pocket infection. Chin J Cardiac Arrhyth. 2003;7(2):100-2.

29. Xia YF, Zhai HX, Li L, Liu RM, Zhang XW, Zhang CH. Effect of antibiotic solution flushing on prevention of pacemaker pocket infection in elderly patients. Chin Heart J. 2007;19(6):705-6.

30. Yang D. Different methods and related risk factor for infection after electronic device implantation: a retrospective analysis of 1659 cases. Kunming: Kunming Medical University; 2015.

31. Zhang WH, Liang YP, Yang J, Jiang L, Feng HL. Influence of intraoperative flushing of pocket with antibiotics on postoperative pocket infections in elderly patients undergoing permanent pacemaker implantation. Chin J Nosocomiol. 2016;26(14):3284-6.

32. Zhou HY, Wang B, Liu MH, Zhang H. Effect of local pocket rinse with antibiotics on peri-operative infection of packmaker pocket. Chin J Prac Nurs. 2010;26(1):29-30

33. Lakshmanadoss U, Nuanez B, Kutinsky I, Khalid R, Haines DE, Wong WS. Incidence of Pocket Infection Postcardiac Device Implantation Using Antibiotic versus Saline Solution for Pocket Irrigation. Pacing Clin Electrophysiol. 2016;39(9):978-84.
34. Mitchell C, David B, William G, Victoria V, Ronn T, Rhodes L. Pediatric pacemaker infections: Twenty years of experience. J Thorac Cardiovasc Surg 2002;124(4):821-7.

35. John F, Qi Z, Di BL, Kevin F, Jay G, Nils G et al. Use and usefulness of antimicrobial pocket irrigation for infection prophylxis in cardiovascular implantable electronic devices. 12th Annual Congress of the European Cardiac Arrhythmia Society.

36. Epstein AE, DiMarco JP, Ellenbogen KA, Estes NA, Freedman RA, Gettes LS, et al. 2012 ACCF/AHA/HRS focused update incorporated into the ACCF/ AHA/HRS 2008 guidelines for device-based therapy of cardiac rhythm abnormalities: a report of the American College of Cardiology Foundation/ American Heart Association Task Force on Practice Guidelines and the Heart Rhythm Society. J Am Coll Cardiol. 2013;61(3):e6-75.

\section{Submit your next manuscript to BioMed Central and we will help you at every step:}

- We accept pre-submission inquiries

- Our selector tool helps you to find the most relevant journal

- We provide round the clock customer support

- Convenient online submission

- Thorough peer review

- Inclusion in PubMed and all major indexing services

- Maximum visibility for your research

Submit your manuscript at www.biomedcentral.com/submit
Biomed Central 